\title{
Localisation of malignant germ-cell tumours by external scanning after injection of radiolabelled anti-alpha-fetoprotein
}

\author{
A K HALSALL, D S FAIRWEATHER, A R BRADWELL, J C BLACKBURN, P W DYKES, \\ A HOWELL, A REEDER, K R HINE
}

\begin{abstract}
Sheep IgG antibody to alpha-fetoprotein was labelled with ${ }^{131} I$ and used to identify human germ-cell tumours by emission scanning. Eleven patients were studied after resection of their primary tumours. Ten had malignant teratoma and one an endodermal sinus tumour. All eight patients with raised serum alphafetoprotein concentrations had metastases apparent in the antibody scans. Of the remaining three patients witir normal serum alpha-fetoprotein concentrations, two had positive scans. Three of the patients with positive results were scanned twice; the second scans were negative after treatment, when the alpha-fetoprotein concentrations had returned to normal.
\end{abstract}

These results suggest that antibody scans are useful in the clinical management of patients with germ-cell tumours.

\section{Introduction}

In recent years much interest has centred on the radioimmunodetection of tumours by external emission scanning. ${ }^{1-3}$ This renewed interest in an old idea has been largely based on the success of subtraction scanning. ${ }^{1}$ Good results have been achieved with the detection of several tumour types producing a variety of protein markers, such as carcinoembryonic antigen, ${ }^{1}$ human chorionic gonadotrophin, ${ }^{4}$ and alpha-fetoprotein. ${ }^{5}$ Attention has focused on carcinoembryonic antigen in gut and breast carcinomas. The reported success rate for detecting primary and secondary tumours is upwards of $75 \% .^{12}$ The specific uptake of radiolabelled antibody into the tumour ranges from two to five times the surrounding normal tissues. ${ }^{3}$ One promising application of the technique is in detecting tumours that produce alpha-fetoprotein. Germ-cell tumours such as malignant teratoma and endodermal sinus tumour, though uncommon, are amenable to chemotherapy even when widely disseminated. ${ }^{6}$ Though alpha-fetoprotein is not a cellsurface marker (being associated with the cytoplasm of yolk-sac

\footnotetext{
Department of Immunology, University of Birmingham Medical School, Birmingham B12 5TJ

A K HALSALL, FRCS, research fellow

D S FAIRWEATHER, MRCP, research fellow

A R BRADWELL, MRCP, senior lecturer

J C BLACKBURN, technician

General Hospital, Birmingham B4 6NH

P W DYKES, MD, FRCP, consultant physician

University Department of Medicine, Birmingham B15 2TH

A HOWELL, MRCP, senior lecturer

Department of Nuclear Medicine, Queen Elizabeth Hospital,
Birmingham B15 2TH

A REEDER, MSC, physicist

Derby Royal Infirmary, Derby DE1 2QY

K R HINE, MRCP, senior registrar
}

elements), it is less heterogeneous than carcinoembryonic antigen and is not widely expressed on normal tissues. Antialpha-fetoprotein might therefore give better localisation than anti-carcinoembryonic antigen. Furthermore, the production of alpha-fetoprotein by these tumours accurately reflects the total tumour burden ${ }^{7}$ in contrast to carcinoembryonic antigen produced by gut tumours.'

Because of the potential benefits of accurate staging both before and after treatment we wished to evaluate the role of antibody emission scanning in patients with germ-cell tumours. This may be particularly useful in distinguishing between malignant and non-malignant masses in patients after chemotherapy, which may not be possible by conventional techniques.

\section{Patients and methods}

We studied 11 patients aged 16-34 years, of whom 10 had malignant teratoma and one an endodermal sinus tumour. Five of the patients with malignant teratoma were studied soon after orchidectomy (group A) as part of their initial investigations, which included bipedal lymphangiography and computed tomography. Three of these were studied again after treatment when thought to be in full remission with normal serum alpha-fetoprotein concentrations. The remaining six patients (group B) were studied up to four years after their initial treatment. One had obvious clinical recurrence and high serum alpha-fetoprotein concentration (case 1). Three patients (cases 4,6 , and 8 ) had raised serum alpha-fetoprotein concentrations which were thought to be due to recurrence of tumour but the sites were not clinically detectable. Two patients (cases 9 and 11) had normal serum alpha-fetoprotein concentrations and were in clinical remission. A twelfth patient with a seminoma was scanned to provide a negative control.

Antiserum to alpha-fetoprotein was prepared in sheep by immunisation with alpha-fetoprotein purified from amniotic fluid. The antiserum was rendered specific by adsorption on glutaraldehydeprecipitated human serum and the IgG fraction purified by ionexchange chromatography. ${ }^{2}$ The antibody was sterilised by passage through a $0 \cdot 2 \mu \mathrm{m}$ filter and tested for toxicity in rabbits. It was then labelled under sterile conditions with ${ }^{131}$ I (chloramine-T method) to a specific activity of about $6 \mu \mathrm{Ci} / \mu \mathrm{g}$ protein. Free iodine was removed on a Sephadex G25 column and the labelled antiserum diluted in isotonic saline containing $1 \%$ human serum albumin. This solution was centrifuged for 12 hours at $20000 \mathrm{~g}$ and filtered again before use.

The concentration of alpha-fetoprotein was measured by radioimmunoassay, in which $1 \mathrm{IU}$ is equivalent to $1.21 \mathrm{ng}$ alpha-fetoprotein. ${ }^{8} \mathrm{~A}$ blood sample for measurement of alpha-fetoprotein was taken immediately before the injection of antibody. The diagnoses of all patients were made by routine histological examination of excised primary tumours.

Scanning procedure-Thyroidal uptake of ${ }^{131} \mathrm{I}$ was blocked by potassium iodide $180 \mathrm{mg}$ daily by mouth, starting 24 hours before the injection of antibody and continued for two weeks. A small bolus of ${ }^{131}$ I-anti-alpha-fetoprotein was given to exclude hypersensitivity to sheep serum (no reactions recorded) followed by approximately $600 \mu \mathrm{Ci}$ labelled antiserum. Scans were recorded 24 hours after the injection and again at 48 hours if the first scan was negative. ${ }^{99 \mathrm{~m}} \mathrm{Tc}$-pertechnetate $(500 \mu \mathrm{Ci})$ and ${ }^{99 \mathrm{~m}} \mathrm{Tc}$-labelled human albumin $(500 \mu \mathrm{Ci})$ were given 30 and five minutes respectively before each scan to delineate the extravascular and vascular spaces. Images of the chest and abdomen were obtained with a Searle LFOV gammacamera fitted with a medium energy, parallel-hole collimato and linked to a DEC PDP $11 / 40$ computer with a dual isotope facility. 
Data were stored and displayed in a $64 \times 64$ matrix and the technetium scan subtracted from the iodide scan after normalising the respective activities over the heart. This subtraction enhanced the image contrast about twofold. All scans were assessed by three of us who had knowledge of the clinical state of the patients and by one of us without this information. There was good agreement between the two assessments.

\section{Results}

The scan was positive in all patients with a raised serum alphafetoprotein concentration (table I) and also in two with normal concentrations but in whom other tests were abnormal. One patient was normal by all tests, as were the three patients who were rescanned after successful treatment. High concentrations of serum alphafetoprotein did not appear to influence the scan result and reduction in tumour burden in three patients was accompanied by a change to a negative scan.

Figure 1 shows the antibody scan and computed tomogram in case 5 before treatment: there was massive enlargement of the para-aortic nodes. He received four courses of chemotherapy with cis-platinium, vinblastine, and bleomycin. One month after the first scan his serum alpha-fetoprotein concentration had returned to normal (and remained so), but after three months of chemotherapy
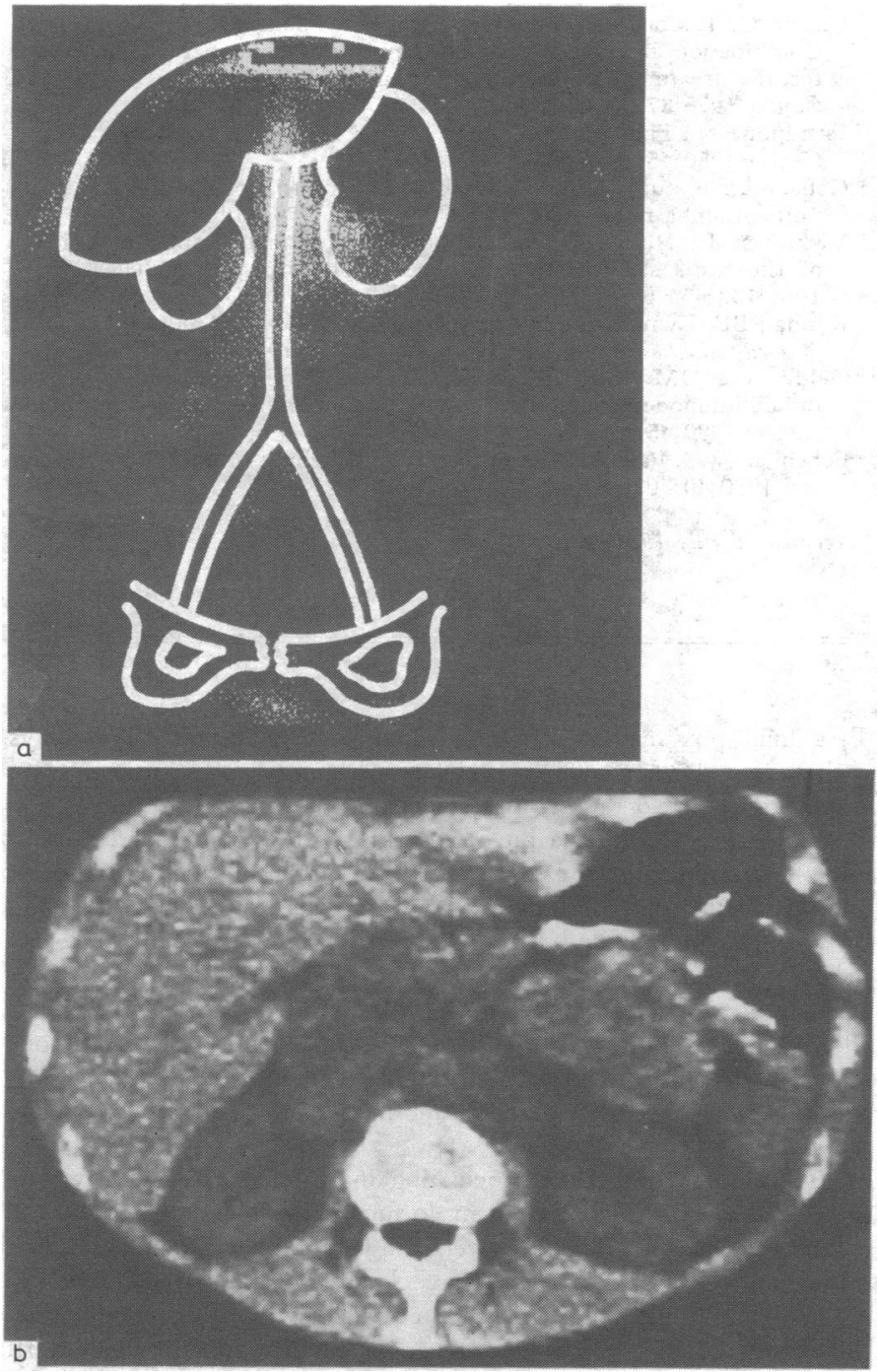

FIG 1-Case 5. Antibody scan and computed tomogram before treatment. (a) Abdominal antibody scan in November 1979. Peripheral ring is artefact of subtraction process. Top corresponds to diaphragm, and bottom to pubis. Central area of emission abnormal. (b) Computed tomogram (with contrast in stomach) in December 1979 showing para-aortic mass corresponding to antibody scan. there was still evidence of residual tumour. Laparotomy was performed and biopsy showed necrotic masses with few surviving malignant cells. The second antibody scan and computed tomogram (fig 2), taken seven months after the first scans, clearly showed the reduction of tumour burden.

Table II shows the 15 sites of secondary deposits in the 10 subjects who had alpha-fetoprotein-producing tumours; 12 were positive on antibody scans. Computed tomography failed to detect five abnormal sites positive on antibody scanning, while lymphangiography missed
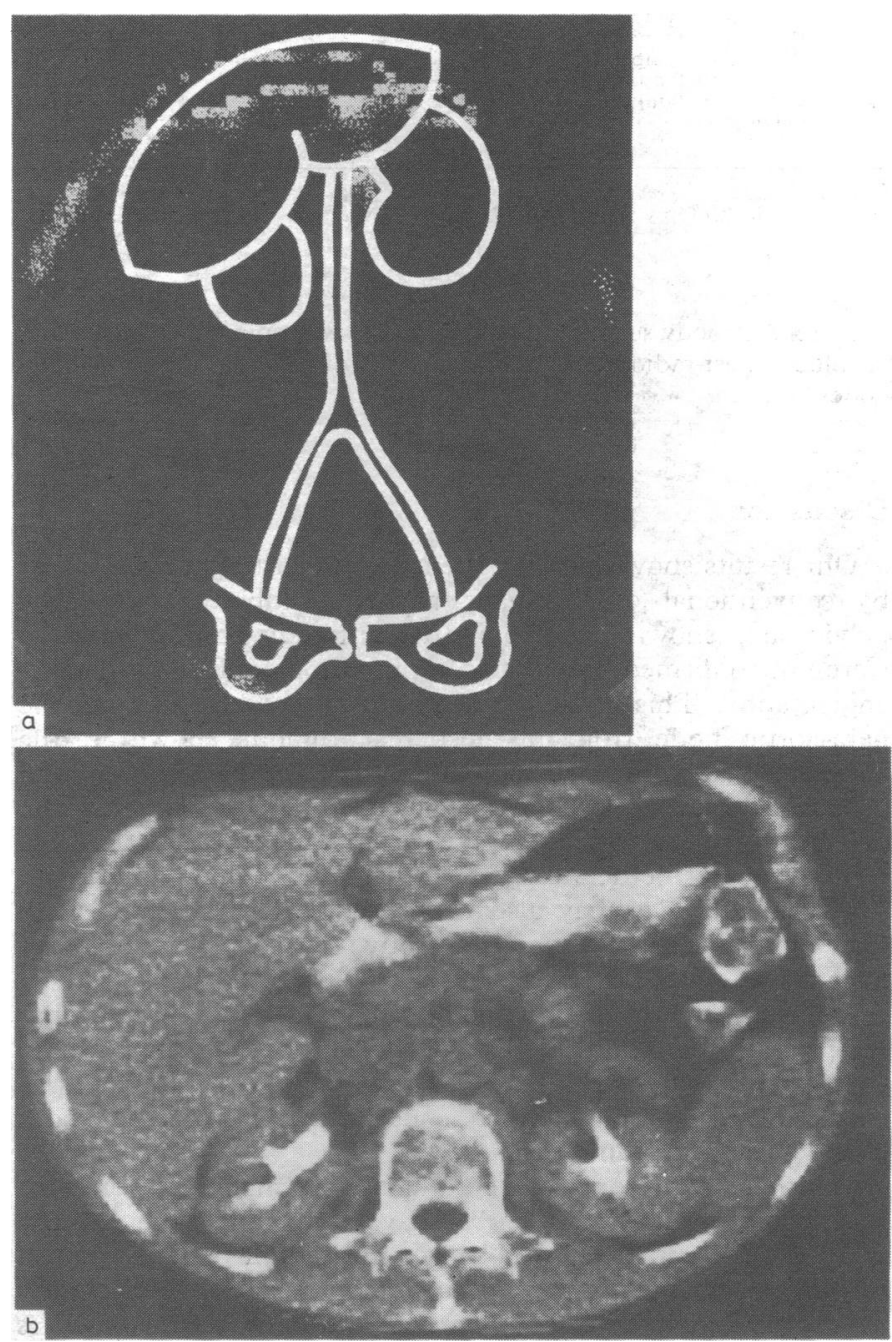

FIG 2-Case 5. Antibody scan and computed tomogram after treatment (a) Abdominal antibody scan in June 1980. Area covered is same as in fig $1(a)$; peripheral ring again artefactual. (b) Computed tomogram in June 1980 (with oral and intravenous contrast). Section at approximately same level as in fig $1(b)$. Scan considered normal.

TABLE I-Antibody scans and serum alpha-fetoprotein concentrations in malignant teratoma

\begin{tabular}{|c|c|c|c|c|c|}
\hline $\begin{array}{l}\text { Case } \\
\text { No }\end{array}$ & \multicolumn{2}{|c|}{ Diagnosis } & $\begin{array}{c}\text { Serum } \\
\text { alpha-fetoprotein } \\
(\mathrm{IU} / \mathrm{ml})\end{array}$ & $\begin{array}{l}\text { Antibody } \\
\text { scan }\end{array}$ & Group \\
\hline 1 & \multicolumn{2}{|c|}{ Malignant teratoma of testis } & 5341 & + & B \\
\hline 2 & " & ", & 1498 & + & A \\
\hline 3 & \multirow{3}{*}{\multicolumn{2}{|c|}{$\begin{array}{l}\text { Endödermal sinus tumour } \\
\text { Malignant teratoma of testis }\end{array}$}} & 1231 & + & A \\
\hline 4 & & & 263 & + & B \\
\hline 5 & & & 187 & + & A \\
\hline 6 & ", & " & 117 & + & B \\
\hline 7 & ", & $"$, & 98 & + & $\mathrm{A}_{\mathbf{B}}$ \\
\hline $\begin{array}{l}8 \\
9\end{array}$ & Mali"gnant terato & of "̈ary & 59 & + & $\mathbf{B}_{\mathbf{B}}$ \\
\hline 9 & Malignant terato & of ovary & $<3$ & + & $\mathbf{B}$ \\
\hline 10 & Malignant terato & of testis & $<3$ & + & A \\
\hline 11 & ” & $"$, & $<3$ & - & B \\
\hline $10^{*}$ & ” & ", ", & $<3$ & - & B \\
\hline $5^{*}$ & ” & $" \Longrightarrow$ & $<3$ & - & $\underset{\mathbf{B}}{\mathbf{B}}$ \\
\hline $3 *$ & "Seminom" & ", ", & $<3$ & - & B \\
\hline $12 \dagger$ & Seminoma & & $<3$ & - & A \\
\hline
\end{tabular}

*Patient scanned twice. †Negative control patient. 
TABLE II-Comparison of antibody scans and conventional tests

\begin{tabular}{|c|c|c|c|c|}
\hline $\begin{array}{l}\text { Case } \\
\text { No }\end{array}$ & Site of deposit & $\begin{array}{l}\text { Antibody } \\
\text { scan }\end{array}$ & $\begin{array}{l}\text { Computed } \\
\text { tomogram }\end{array}$ & Lymphangiogram \\
\hline 1 & Vertebral & + & + & \pm \\
\hline 2 & Liver & + & \pm & $\pm^{+}$ \\
\hline 3 & Para-aortic lymph node & + & + & + \\
\hline 3 & Pulmonary & - & ++ & \\
\hline 4 & Pelvic & + & $-*$ & ND \\
\hline & Para-aortic lymph node & + & + & ND \\
\hline 5 & Pulmonary & - & + & $\stackrel{+}{+}$ \\
\hline 6 & $\begin{array}{l}\text { Lett supraclavicular lymph node } \\
\text { Para-aortic lymph node }\end{array}$ & $\bar{t}$ & + & ND \\
\hline 7 & Para-aortic lymph node & + & + & + \\
\hline 8 & Para-aortic lymph node & + & + & ND \\
\hline 9 & Para-aortic lymph node & + & - & + \\
\hline 10 & $\left\{\begin{array}{l}\text { Para-aortic lymph node } \\
\text { Pelvic }\end{array}\right.$ & + & $\overline{-}$ & + \\
\hline
\end{tabular}

$\mathrm{ND}=$ Not done

*Positive at laparotomy. †Palpable. $¥$ Not appropriate.

one site. Antibody scanning failed to detect two pulmonary metastases visible in chest radiographs and one group of palpable supraclavicular nodes.

\section{Discussion}

Our results show that all patients who had tumour diagnosed by conventional criteria gave positive antibody scans. The pelvic sites shown positive in the antibody scan in case 10 were not confirmed by either computed tomography or lymphangiography. This patient did, however, have deposits in the para-aortic node from his testicular tumour shown in the antibody scan, computed tomogram, and lymphangiogram, and probably lower nodes also contained tumour. Lateral aortic nodes, which are the first site of tumour spread from the testes, may not be delineated by pedal lymphangiography, ${ }^{9}$ and some pelvic nodes may also be missed. ${ }^{10}$ We therefore think that there were no true false-positive results, and the falsenegative rate was low.

Despite the theoretical objection that circulating alphafetoprotein might inhibit the injected antibody from reaching the tumour, successful scans were obtained in patients with very high circulating antigen concentrations. This has been found with other tumour markers. ${ }^{2}$

In other studies only three patients with malignant teratoma have had antibody scans. ${ }^{511}$ We have studied 11 such patients and have rescanned three. They comprised all patients seen in our unit over nine months and covered a wide range of clinical problems. This type of imaging seems to be at least as good as a combination of conventional techniques and is probably more sensitive. The method allows an assessment after surgical clearance, which may be useful if the rate of fall of the alphafetoprotein concentration suggests incomplete removal of tumour. Furthermore, a positive scan indicates malignant tissue, whereas other techniques are less specific.

Antibody scans, however, are not as well defined as computed tomograms or conventional radioisotope imaging, and some skill is required when assessing the images around the bladder and stomach, as both sites concentrate free iodine. An alternative isotope might avoid this problem. We have not investigated patients with primary hepatomas because the results would not be therapeutically useful, but a previous study showed positive scans in such patients. ${ }^{5}$

The resolution of this method depends on the imaging equipment and in our hands is at present about two centimetres. We may well, however, detect lesions much smaller than this, as the sensitivity depends on the increased emission from the tumour compared with surrounding normal tissue. The latter may be improved with different isotopes ${ }^{12}$ and adding computed tomography to the scanning procedure.

We thank the radiotherapy consultants at the Queen Elizabeth Medical Centre; Mr I Scott, Derbyshire Hospital for Women; and
Dr W G Jones, Cookridge Hospital, Leeds, for allowing us to study their patients; and Dr A Milford-Ward, department of immunology, University of Sheffield, who carried out the alpha-fetoprotein assays.

Dr S Fairweather, Dr A K Halsall, and Miss J C Blackburn are supported by a grant from the Cancer Research Campaign. Dr K Hine was supported by the Medical Research Council.

Requests for reprints should be addressed to: Dr D S Fairweather, Immunodiagnostic Research Laboratory, Department of Immunology, University of Birmingham Medical School, Birmingham B15 2TJ.

\section{References}

${ }^{1}$ Goldenberg DM, Deland F, Kim EE, et al. Use of radiolabelled antibodies to carcinoembryonic antigen for the detection and localisation of diverse cancers by external photoscanning. $N$ Engl $f$ Med 1978;298: 1384-8.

2 Dykes PW, Hine KR, Bradwell AR, et al. Localisation of tumour deposits by external scanning after injection of radiolabelled anti-carcinoembryonic antigen. $\mathrm{Br}$ Med $\mathcal{f} 1980 ; 280: 220-2$.

${ }^{3}$ Mach J-P, Carrel S, Forni M, Ritschard J, Donath A, Alberto P. Tumor localisation of radiolabelled antibodies against carcinoembryonic antigen in patients with carcinoma, a critical evaluation. $N$ Engl f Med 1980;303:5-10.

${ }^{4}$ Bagshaw KD, Searle F, Lewis J, Brown P, Keep P. Preliminary therapeutic and localisation studies with human chorionic gonadotrophin. Cancer Res 1980;40:3016-7.

${ }^{5} \mathrm{Kim}$ EE, Deland FH, Nelson MD, et al. Radioimmunodetection of cancer with radiolabelled antibodies to alpha-fetoprotein. Cancer Res 1980; 40:3008-12.

${ }^{6}$ Merrin C, Takita H, Weber R, Wajsman Z, Baumgartmer J, Murphy J. Combination radical surgery and multiple sequential chemotherapy for the treatment of advanced carcinoma of the testis (stage III). Cancer $1976 ; 37: 20-9$.

${ }^{7}$ Javadpour $\mathrm{N}$. The role of biologic tumor markers in testicular cancer. Cancer $1980 ; 45: 1755-61$.

${ }^{8}$ Collaborative study. Equivalence between international units and mass units of alpha-fetoprotein. Clin Chim Acta 1979;96:59-65.

${ }^{9}$ Wahlqvist L, Hulten L, Rosencrantz $M$. Normal lymphatic drainage of the testis studied by funicular lymphography. Acta Chir Scand $1966 ; 132: 454-65$.

${ }^{10}$ Kendall BE. Lymphography. In: Shanks SC, Kerley P, eds. A text-book of $x$-ray diagnosis. London: HK Lewis \& $\mathrm{Co}, 1972: 594-655$.

${ }^{11}$ Goldenberg DM, Kim EE, Deland F, et al. Clinical studies on the radioimmunodetection of tumors containing alpha-fetoprotein. Cancer $1980 ; 45: 2500-5$.

${ }^{12}$ Eckelman WC, Paik CH, Reba RC. Radiolabelling of antibodies. Cancer Res $1980 ; 40: 3036-42$.

(Accepted 16 fuly 1981)

THE fmall-pox may be communicated in a great variety of ways with nearly the fame defree of fafety and fuccefs. In Turkey, from whence we learned the practice, the women communicate the difeafe to children, by opening a bit of the fkin with a needle, and putting into the wound a little matter taken from a ripe puftule. ;n the coaft of Barbary they pafs a thread wet with the matter through the fkin, between the thumb and fore-finger; and in fome of the ftates of Barbary, inoculation is performed by rubbing in the variolous matter between the thumb and fore-finger, or on other parts of the body. The practice of communicating the fmall-pox, by rubbing the variolous matter upon the fkin, has been long known in many parts of Afia and Europe, as well as in Barbary, and has generally gone by the name of buying the Small-pox.

THE prefent method of inoculating in Britain is to make two or three flanting incifions in the arm, fo fuperficial as not to pierce quite through the fkin, with a lancet wet with frefh matter taken from a ripe puftule; afterwards the wounds are clofed up, and left without any dreffing. Some make ufe of a lancet covered with the dry matter; but this is lefs certain, and ought never to be ufed unlefs where frefh matter cannot be obtained: when this is the cafe, the matter ought to be moiftened by holding the lancet for fome time in the fteam of warm water.

INDEED, if frefh matter be applied long enough to the fkin, there is no occafion for any wound at all.

(Buchan's Domestic Medicine, 1786.) 